\title{
Evolution des types cellulaires du lait de brebis (race Churra) en fonction des dénombrements cellulaires totaux pendant la traite mécanique et manuelle
}

\author{
C. GONZALO et V.R. GAUDIOSO \\ Departamento de Producción Animal \\ Facultad de Veterinaria. Campus «La Palomera», León (España)
}

\begin{abstract}
Résumé
L'évolution du nombre total et du pourcentage des types cellulaires (polymorphonucléaires, macrophages, agranulocytes et autres) en fonction des dénombrements cellulaires totaux est étudiée dans le lait de 91 brebis de race Churra : 42 soumises à la traite mécanique et 49 avec traite manuelle.

Ce travail montre que : a) Les pourcentages de chaque type cellulaire subissent de fortes variations au fur et à mesure que la concentration cellulaire totale du lait augmente. L'analyse des courbes de régression présente des coefficients de corrélation très significatifs $(p<0,01)$. b) Les concentrations absolues de chaque type cellulaire suivent des ajustements linéaires de pente positive et des coefficients de corrélation très significatifs $(p<0,01)$, aussi bien lors de la traite mécanique que manuelle, c) Le nombre et la proportion des principaux types cellulaires présents dans le lait (polymorphonucléaires, macrophages et agranulocytes) ne semblent pas dépendre du mode de traite.
\end{abstract}

Au-dessus des 500000 cellules $/ \mathrm{ml}$ plus de 85 p. 100 des cellules sont des leucocytes (polymorphonucléaires et agranulocytes).

Mots clés : Brebis, lait, mammite, cellules, traite.

\section{Introduction}

Les travaux effectués sur le dénombrement cellulaire dans le lait de brebis, et, en particulier, ceux qui font référence à un seuil au-delà duquel on admet l'existence de mammites subcliniques, sont peu abondants et souvent contradictoires. Ainsi, Zrv et al. (1968) trouvent des réactions négatives au CMT (California Mastitis Test) dans du lait avec des concentrations de plus de $10^{6}$ cellules $/ \mathrm{ml}$; Filev (1972) établit le niveau moyen vers $10^{5}$ cellules/ml ; Vitkov (1979) trouve que 85 p. 100 des brebis échantillonnées ont une numération cellulaire supérieure à $10^{5}$ cellules $/ \mathrm{ml}$, et GrEEN (1984) recommande l'analyse bactériologique du lait dont la concentration dépasse le million de cellules/ml. 
D'autre part il existe une grande diversité de critères quant à la proportion et la signification des différents types cellulaires du lait provenant de mamelles saines (Schalm et al., 1971 ; Lee \& Outteridge, 1981 ; Outteridge \& Lee, 1981 ; Dulin et al., $1983 \mathrm{~b}$ ), sans que la variation des catégories cellulaires au cours de l'inflammation n'ait réellement été abordée.

Ce travail se propose de rapporter l'évolution des différents types cellulaires du lait de brebis en fonction de l'accroissement du niveau cellulaire total; il essaiera de relier ceux-ci au système de traite utilisé.

\section{Matériel et méthodes}

L'étude des types cellulaires est réalisée pour chaque animal entre les 45 et $60^{\circ}$ jours ( $2^{\circ}$ mois de traite) après la parturition. Les échantillons sont prélevés à partir de tout le lait individuel (soigneusement mélangé) de la traite du matin, obtenu sur 91 brebis de race Churra : 42 appartenant à une exploitation avec traite mécanique et 49 avec traite manuelle.

Les animaux sont traits deux fois par jour (le matin à $8 \mathrm{~h}$ et le soir à $18 \mathrm{~h}$ ).

La méthode de traite mécanique est la pose simple des gobelets terminée par un égouttage-retrait de ceux-ci $\left(2^{\circ}\right.$ Symposium International sur la Traite des Petits Ruminants, 1978). La traite manuelle est effectuée " au pouce " (GALTIER, 1943 ; CALCEDO, 1964).

La méthodologie suivie pour la numération et l'identification cellulaire est celle décrite par Thieulin \& Vuillaume (1967) légèrement modifiée (temps d'action du xylol : $8 \mathrm{~min}$; coloration : hematoxiline-eosine).

L'identification cellulaire est uniquement réalisée par critères morphologiques. Suivant LEE et OuTTERIDGE (1981) nous avons distingué les catégories cellulaires suivantes : polymorphonucléaires, macrophages et agranulocytes, auxquelles nous avons ajouté un groupe de cellules difficile à caractériser et/ou dégénérées, que nous incluons sous l'appellation « autres » cellules.

\section{Résultats}

Les pourcentages relatifs de chaque type cellulaire subissent de grandes modifications au fur et à mesure que la concentration cellulaire totale du lait augmente (fig. $n^{\circ} 1$ et 3). En effet, pour les brebis traites à la machine, l'accroissement de cette dernière se traduit par une élévation logarithmique $(r=0,88)$ du pourcentage des polymorphonucléaires, tandis que pour les autres types cellulaires on observe une diminution logarithmique du pourcentage de macrophages $(\mathrm{r}=-0,82)$ et des « autres " cellules $(r=-0,61)$ et une réduction linéaire des agranulocytes $(r=-0,50)$. 


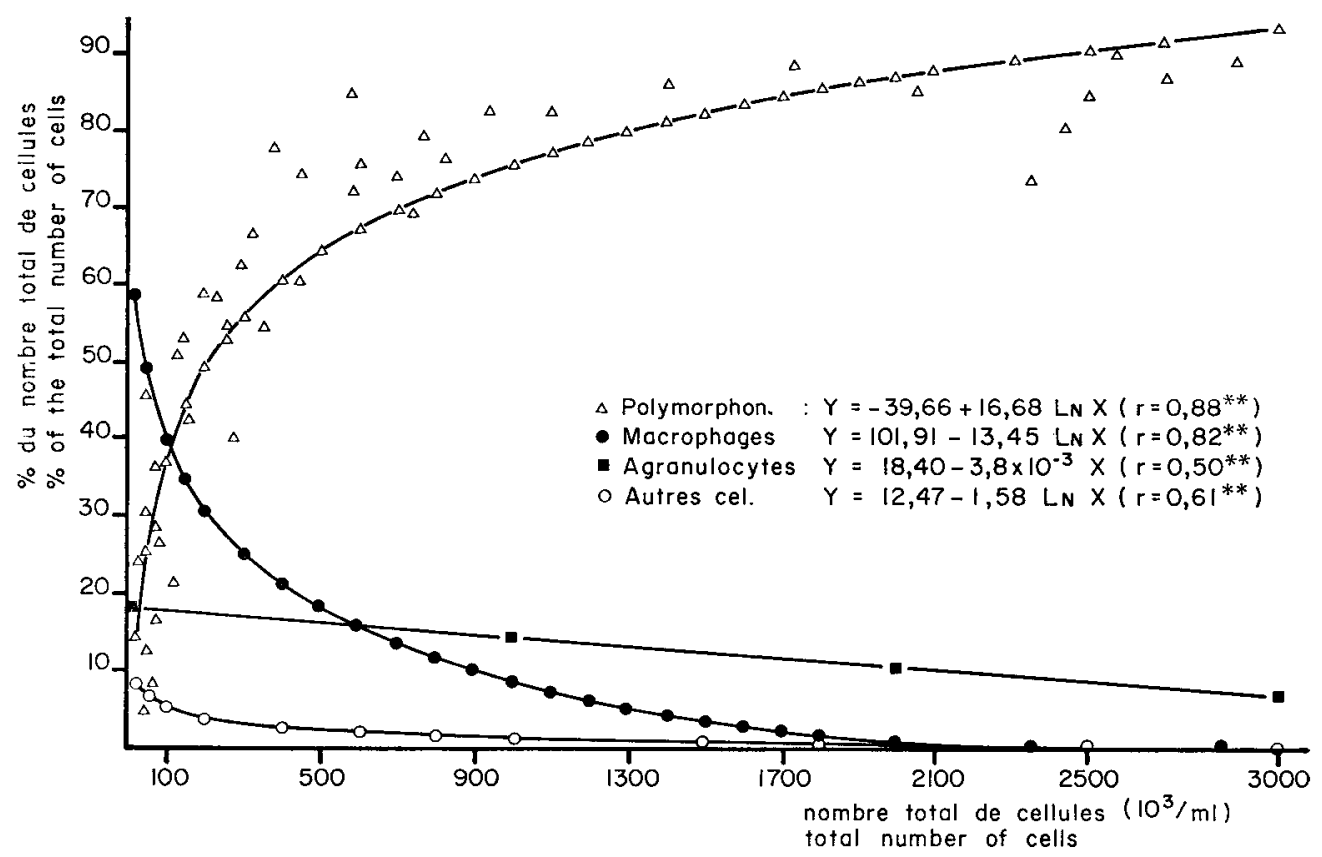

FIG. 1

Evolution du pourcentage des différents types cellulaires trouvés dans le lait de traite mécanique de brebis de race Churra, en fonction des concentrations totales de cellules $(* * p<0,01)$.

Variation in the percentage of the different cell types in the milk of ewes (Churra breed) subjected to machine milking according to total cell concentrations (** $p<0.01)$.

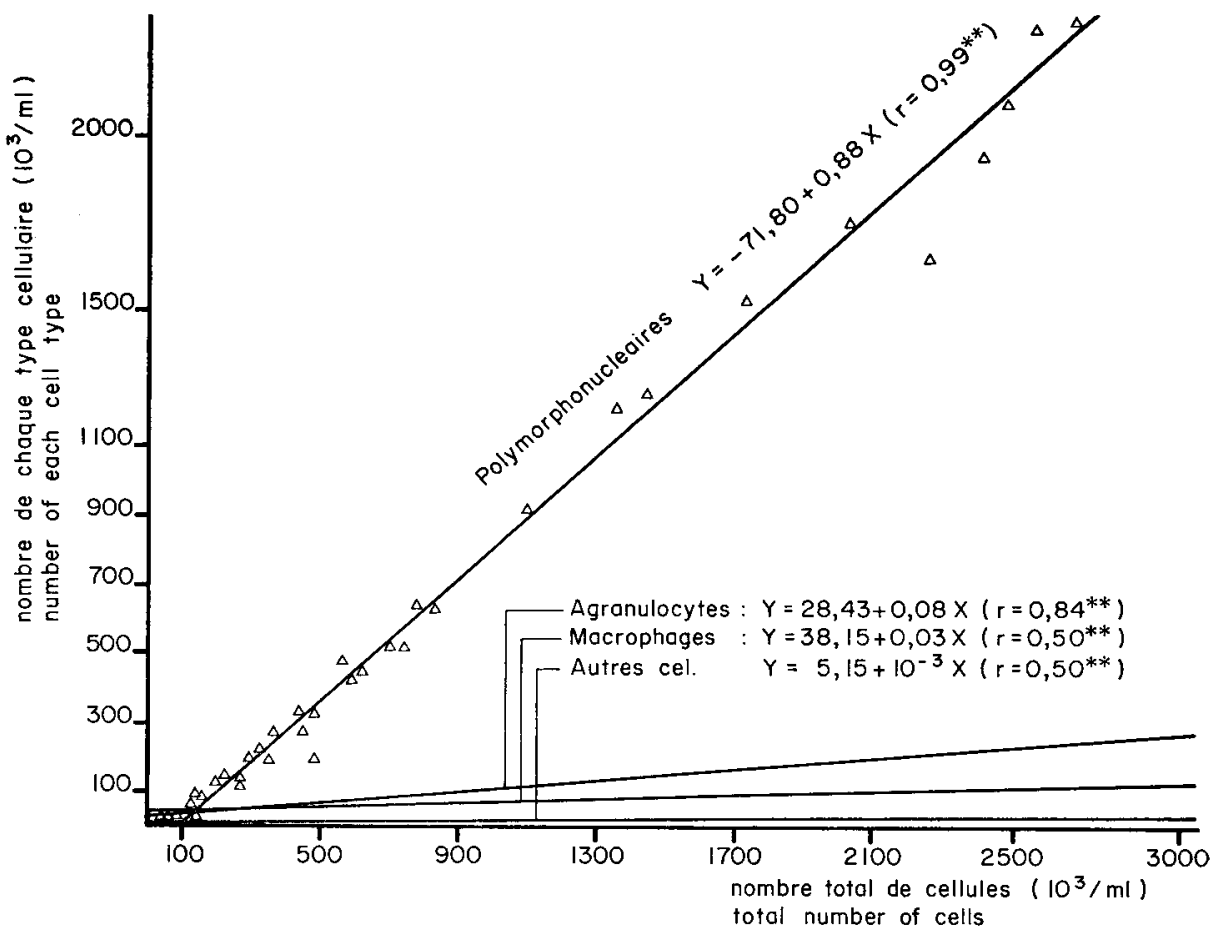

FIG. 2

Evolution des concentrations des types cellulaires du lait de traite mécanique, en fonction des valeurs totales de cellules $(* * 0<0,01)$.

$V$ ariation in the concentrations of the cell types in milk from machine milking according to the total number of cells (*:* $p<0.01)$. 


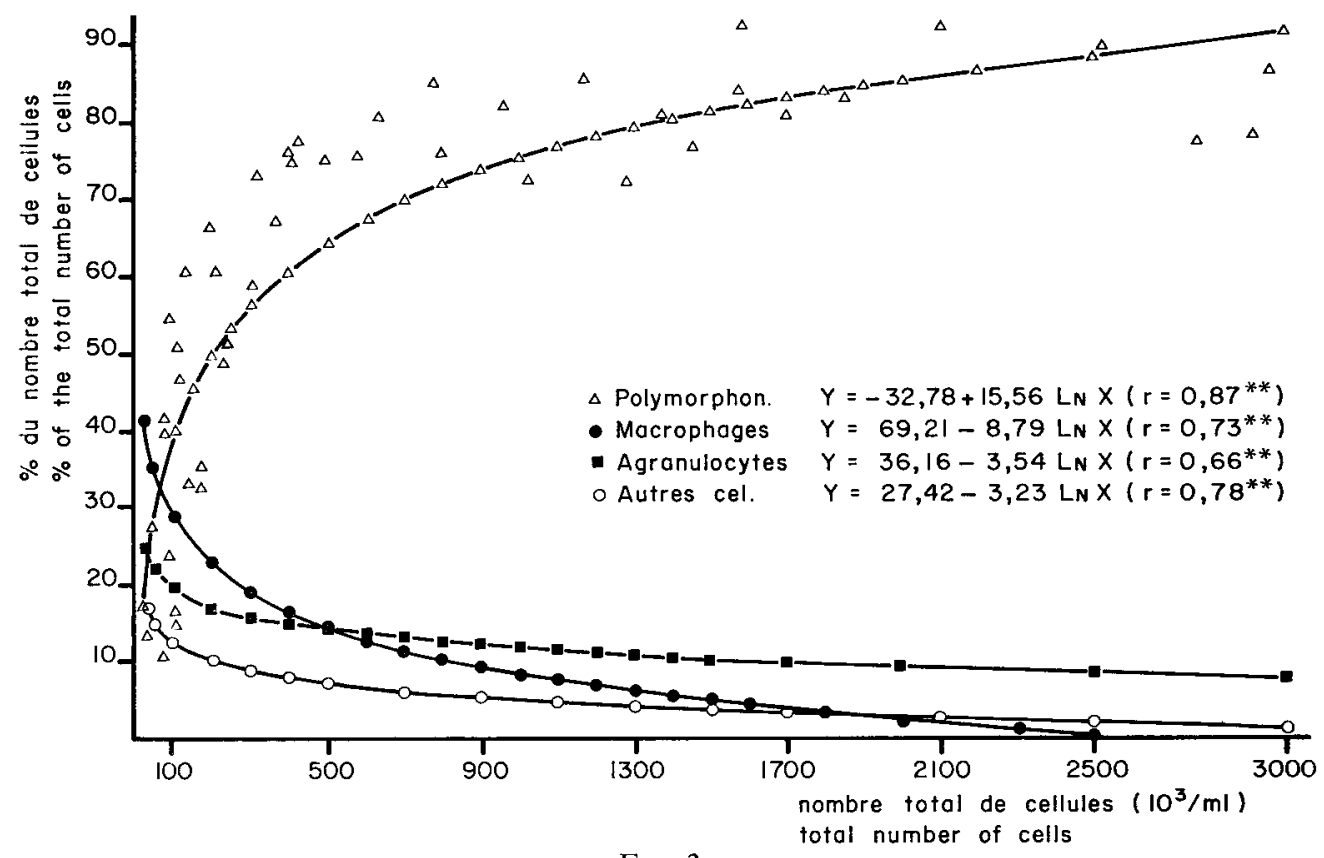

Fig. 3

Evolution du pourcentage des types cellulaires du lait de traite manuelle, en fonction des concentrations totales de cellules (*** $p<0,01)$.

$V$ ariation in the percentage of the cell types in milk from hand milking, according to the total cell concentrations $(* * p .<0.01)$.

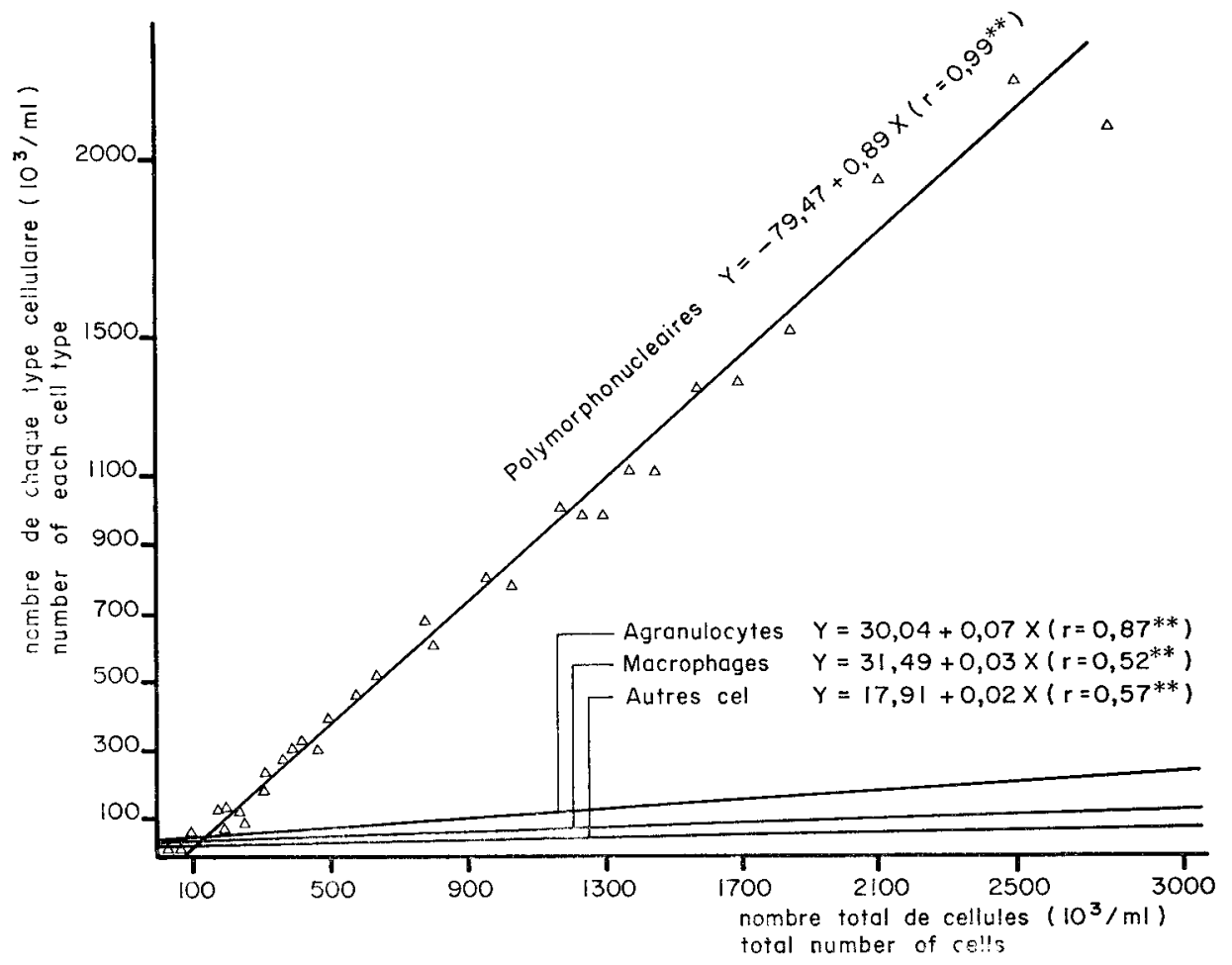

Fig. 4

Evolution des concentrations des différents types cellulaires du lait de traite manuelle, en fonction des concentrations totales de cellules (*** $p<0,01)$.

Variation in the concentrations of the cell types in milk from hand milking, according to the total cell concentrations $(* * x<0.01)$. 
Dans le cas de la traite manuelle, la réponse cellulaire est similaire à celle exposée pour la traite mécanique : les coefficients de corrélation avec la concentration totale sont de $+0,87$ pour les polymorphonucléaires ; $r=-0,73$ pour les macrophages et

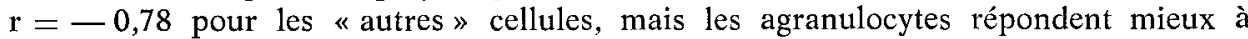
un ajustement logarithmique $(\mathrm{r}=-0,66)$ que linéaire $(\mathrm{r}=-0,55)$. Tous ces coefficients de corrélation sont statistiquement significatifs $(\mathrm{p}<0,01)$.

Les figures 2 et 4 montrent que : a) Les concentrations absolues de chaque type cellulaire suivent des ajustements linéaires de pente positive et des coefficients de corrélation très significatifs $(\mathrm{p}<0,01)$, aussi bien lors de la traite mécanique ( $\mathrm{r}$ entre 0,50 et 0,99 ) que manuelle ( $r$ entre 0,52 et 0,99 ). b) Les pentes des droites des deux types de traite ne sont pas significativement différentes pour les polymorphonucléaires, macrophages et agranulocytes (fig. $\left.n^{\circ} 5\right)$. Le seuil $(p<0,05)$ est par contre atteint pour les « autres» cellules.

Nous n'avons pas trouvé de différences significatives pour le nombre total de cellules selon le type de traite : $757,92 \pm 134,75 \times 10^{3}$ cellules $/ \mathrm{ml}$ pour les brebis traites à la machine, et $946,69 \pm 173,21 \times 10^{3}$ pour les brebis avec traite manuelle.

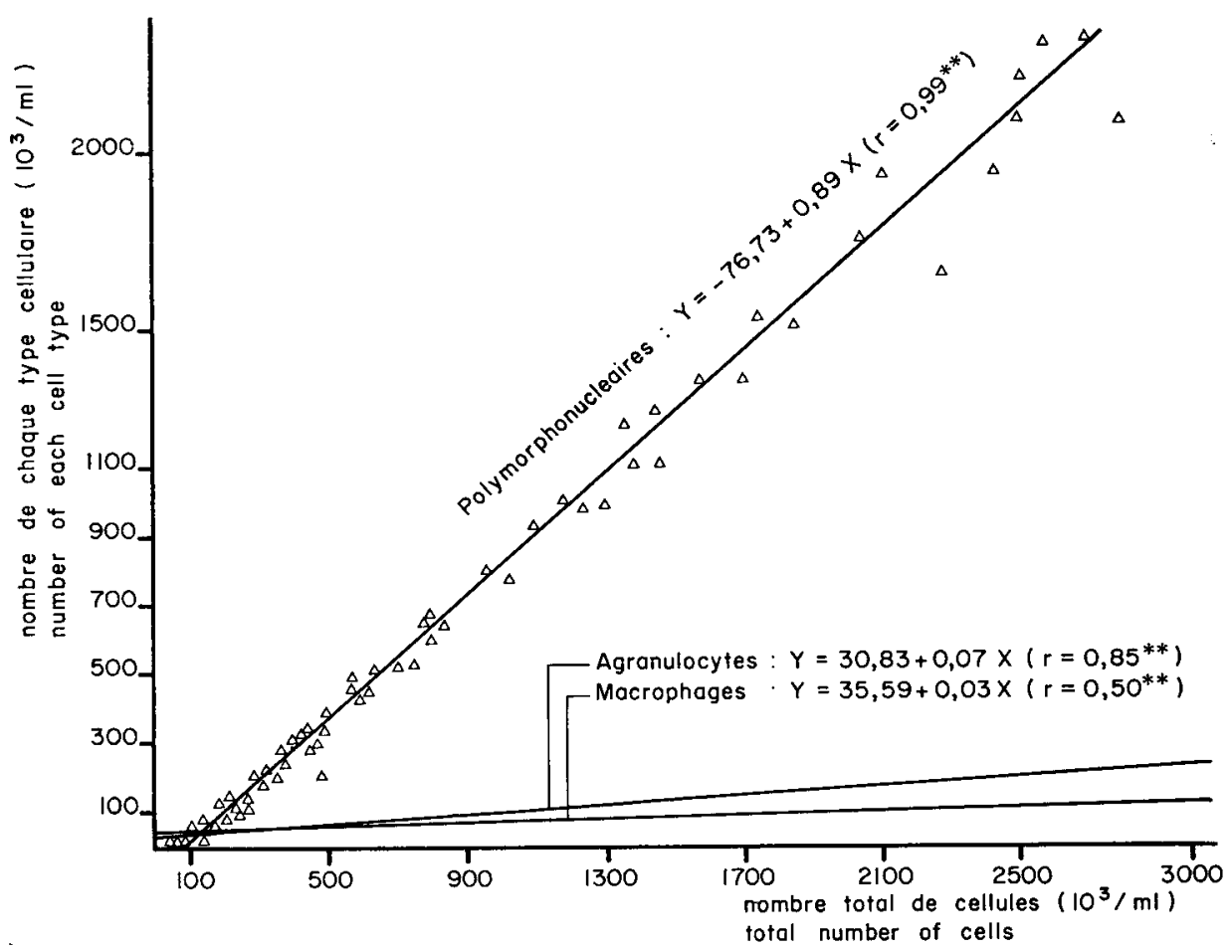

FIG. 5

Evolution des concentrations des types cellulaires

en fonction des numérations totales de cellules

(le graphique regroupe le lait de traite mécanique et manuelle) $(* * p<0,01)$.

$V$ ariation in the concentration of the cell types according to cell total counts

(figure indicates together milk from hand and machine milking) (***; $p<0.01)$. 


\section{Discussion - Conclusion}

Le nombre et la proportion des principaux types cellulaires présents dans le lait de brebis (polymorphonucléaires, macrophages et agranulocytes), varient en fonction de la concentration cellulaire (fig. $n^{\circ} 1,2,3$ et 4 ) d'une façon très semblable pendant la traite mécanique et manuelle. Ceci semble indiquer que la réponse physiopathologique de la mamelle est indépendante du système de traite (fig. $\mathrm{n}^{\mathrm{o}} 5$ ).

L'observation de ces figures révèle d'abord que pour une concentration inféférieure à 100000 cellules/ml (considérée par FILEV, 1972, comme reflétant un excellent état sanitaire du pis) le type cellulaire prédominant est le macrophage ( $\mathrm{a}=101,9$ et 69,2 respectivement pour la traite mécanique et manuelle), suivi par l'agranulocyte $(a=18,4$ et 36,2$)$ et en dernier lieu par le polymorphonucléaire $(\mathrm{a}=-39,7$ et - 32,8). Ces résultats sont globalement en accord avec ceux de LEE \& OUTTERIDGE (1981) et Dulin et al. (1983 a) obtenues avec du lait de brebis allaitantes et saines, puisque pour ces auteurs les macrophages représentent également le type cellulaire principal (70 à 86 p. 100), devant les lymphocytes (10 à 17 p. 100) et les polynucléaires ( 2 à 22 p. 100). Nous remarquerons toutefois que nos pourcentages sont inférieurs pour les macrophages $(45,5 \pm 4,2$ p. 100), supérieurs pour les polymorphonucléaires $(27,9 \pm 3,5$ p. 100$)$ et similaires pour les agranulocytes $(16,9 \pm 1,6$ p. 100). Ces différences peuvent d'une part s'expliquer par le fait que LeE \& OUTTERIDGe (1981) et Dulin et al. (1983 a) n'utilisaient pas le lait total de la traite mais seulement quelques fractions, et d'autre part que les brebis choisies par ces auteurs étaient uniquement allaitantes et n'avaient pas été soumises à la traite mécanique ou manuelle.

Au-dessus de 100000 cellules/ml une croissance rapide du pourcentage des polymorphonucléaires se produit au détriment des macrophages et des agranulocytes. Ceci confirme le rôle prépondérant des polymorphonucléaires dans le développement de la réaction inflammatoire du pis. Les macrophages n'y participeraient pas malgré leur fonction éminemment phagocytaire (LEE \& OuTTERIDGE, 1981), et seraient plutôt révélateurs d'un bon état sanitaire de la mamelle.

Quant aux « autres » cellules (dégénérées et/ou difficiles à classer) on observe des différences significatives $(p<0,05)$ de concentration entre traite mécanique et manuelle. Ce fait est explicable si on les considère comme cellules épithéliales dégénérées et desquamées des acinus glandulaires dont l'obtention pourrait être favorisée par la traite manuelle. Quoi qu'il en soit, les valeurs relatives de cette catégorie cellulaire sont peu importantes.

D'après les résultats obtenus par ScHALm et al. (1971) on pourrait considérer que 300000 cellules $/ \mathrm{ml}$ représente le seuil au-delà duquel la brebis est atteinte de mammite. Ces valeurs ont été confirmées récemment par Tyszka et al. (1984) bien que TravniceK et al. (1984) recommandent 500000 cellules $/ \mathrm{ml}$ comme niveau de différenciation entre le lait normal et celui provenant de pis infectés.

Dans notre étude, lorsque le niveau cellulaire est supérieur à 500000 cellules $/ \mathrm{ml}$, 85 p. 100 des cellules sont des leucocytes (polymorphonucléaires et agranulocytes), ce qui permet de conclure avec certitude à une infection mammaire (LE GALL \& Plommet, 1965 ; Jasper, 1980 ; Lee \& Outteridge, 1981 : Poutrel \& Rainatd, 1982 ; Poutrel, 1983 ; Dulin et al., 1983 b). Dans le même ordre d'idée Vitkov et 
Vitanov (1980) détectent la présence d'une forte proportion d'échantillon contenant Staphylococcus epidermidis et $S$. aureus dans du lait de brebis contenant plus de 500000 cellules $/ \mathrm{ml}$.

Quant au niveau de 300000 cellules/ml, mentionné auparavant, il nous faut constater qu'il correspond selon nos résultats, à 190000 polymorphonucléaires $/ \mathrm{ml}$, qui selon Thieulin \& Vuillaume (1967) et Schalm et al. (1971) différencie chez la vache et chez la brebis le lait normal et mammiteux.

Ces résultats contrastent avec ceux que nous avions obtenus dans les laits de troupeaux de la Région du Duero soumis à la traite mécanique $\left(1600 \times 10^{3}\right.$ cellules/ ml) (Gonzalo \& Gaudioso, 1984). Deux explications peuvent être avancées : 1) le temps de présence des gobelets sur les pis atteignent dans ces exploitations des valeurs élevées $(3,59 \mathrm{~min}$. en moyenne) (Gonzalo et al., 1985) ; 2) la pratique généralisée dans ces troupeaux de l'accouplement continu. En effet, l'application de temps de traite identiques et longs à des brebis récemment sevrées et à celles proches du tarissement ne peut que favoriser l'éclosion de mammites et d'un nombre important de cellules par millilitre.

\section{Remerciements}

A Monsieur le Professeur J. Labussiere qui a bien voulu juger ce travail en nous dispensant son aide et ses conseils.

\section{Summary \\ Variation in sheep milk cell types according to total cell counts in machine and hand milking}

Variation in the total number and percentage of cell types (polymorphonuclear, macrophages, agranulocytes, and others) according to total cell counts was studied in milk from 91 ewes of the Churra breed subjected to hand milking (42) or to machine milking (49).

The following results were obtained :

a) The relative percentages of each cell type varied considerably as the total cell concentration of the milk increased (fig. 1 and 3). Analysis of the regression curves indicated very significant coefficients of correlation $(\mathrm{p}<0.01)$.

b) The absolute concentrations of each cell type followed linear adjustments on an upward slope and showed very significant coefficients of correlation $(p<0.01)$ both in hand and machine milking (fig. 2 and 4).

c) The number and proportion of the main cell types present in milk were independent of the milking method (fig. 5).

Over 500000 cells $/ \mathrm{ml}$, more than 85 per cent cells were leucocytes (polymorphonuclear and agranulocytes).

Key words : Sheep, milk, mastitis, cells, milking.

Reçu en décembre 1984.

Accepté en avril 1985. 


\section{Références bibliographiques}

Calcedo V., 1964. El factor hombre en los rebaños lecheros. Ordeño mecánico en ovinos? Avances en Alimentución y Mejora Animal, 5, 669-675.

Dulin A.M., PaApe M.J., Wergin W.P., 1982. Differenciation and enumeration of somatic cells in goat milk. J. Food Prot., 45, 435-439.

Dulin A.M., Paape M.J., Berkow S., Hamosh M., Hamosh P., 1983 a. Comparison of total somatic cells and differential cellular composition in milk from cows, sheep, goats and human. Dairy Sci. Abstr., 45, 908.

Durin A.M., PaApe M.J., Schultze W.D., Weinland B.T., 1983 b. Effect of parity, stage of lactation, and intramammary infection on concentration of somatic cells and cytoplasmic particles in goat milk. J. Dairy Sci., 66, 2426-2433.

FileV F., 1972. Microbiologic studies of ewe's milk. VI. Cell conte. Vet. Nauki Sofia, 5, 27-32.

iGaltier L., 1943. La Traite des Brebis. Leur lait et le fromage de Roquefort. Thèse. Ecole Nat Vét. Lyon. Faculté de Méd. et de Pharm. de Lyon. 167 p.

Gonzalo C., Gaudioso V.R., 1984. Recuento celular en leche de oveja. Comparación entre ordeño mecánico y manuel. $3^{e r}$ Symposium Internacional de Ordeño Mecánico de Pequeños Rumiantes. Valladolid (España), 268-275.

Gonzalo C., Gaudroso V.R., Sotillo J.L., 1985. Organización del trabajo de ordeño en las explotaciones comerciales de ganado ovino. Zootechnia, XXXIII, 268-273.

Green T.J., 1984. Use of somatic cell counts for detection of subclinical mastitis in ewes. Vet. Rec., 114, 43-44.

JASPER D.E., 1980. Mastitis. In : Bovine Medicine and Surgery. Vol. II, 1047-1093 (2nd ed.). Ed. H.E. Amstutz. American Veterinary Publ. Inc. U.S.A.

Le Gall A., Plommet M., 1965. Observations sur la croissance des staphylocoques et la réaction leucocytaire au cours des premières heures de la mammite expérimentale de la brebis. Ann. Biol. Anim Biochim. Biophys., 5, 113-130.

LeE C.S., OutTeridge P.M., 1981. Leucocytes of sheep colostrum, milk and involution secretion, with particular reference to ultrastructure and lymphocyte sub-populations. J. Dairy Res., 48, 225-237.

OUtTERidge P.M., LEE C.S., 1981. Cellular immunity in the mammary gland with particular reference to $\mathrm{T}, \mathrm{B}$ lymphocytes and macrophages. The Ruminant Immutne System. Ed. J.E. Butler. Plenum Publishing Corporation. U.S.A., 513-534.

Poutrel B., Rainard P., 1982. Predicting the probability of quarter infection (by major pathogens) from somatic cell concentration. Am. J. Vet. Res., 43, 1296-1299.

Poutrel B., 1983. Les mammites de la chèvre et de la brebis. Les Dossiers de l'Elcuage, $5,37-45$.

Schalm O.W., Carrol B.S., Jain N.C., 1971. Bovine Mastitis, Lea and Febiger, Philadelphia. U.S.A., 94-157.

Thileulin G., Vuillaume R., 1967. Eléments pratiques d'analyse et d'inspection du lait de produits laitiers et des œufs. Le Lait ( $n^{\prime \prime}$ hors série), 160-173.

Traynicek M., Petrik P., Federic F., Balascak J., 1984. Diagnóstico de las mamitis en las ovejas mediante el conteo celular electrónico. $3^{e}$ Symposium Internacional de Ordeño Mecánico de Pequeños Rumiantes. Valladolid (España), 499-505.

Tyszka Z., Skolasinski W., ZarzyCKi J., Malgorzata K., 1984. Various factors, genetic in particular, causing mastitis in sheep. 3rd Symposium Internacional de Ordeño Mecánico de Pequeños Rumiantes. Valladolid (España), 529-538.

VıTKov M., 1979. Study of subclinical mastitis in machine milked ewes. Veterinarnomeditsinski Nauki, 16, 66-71.

VitKov M., Vitanov S., 1980. Cell content of ewes'milk in machine milking. Veterinarnomeditsinski Nauki, 17, 53-58.

Ziv G., Shacked A., Risenberg-Tirer R., 1968. The effectiveness of the California mastitis test as a mesure of somatic cell counts of ewe's milk. Refuah Veterinarith, 25, 179-184. 